\title{
Adverse events and preventive measures related to COVID-19 vaccines
}

\author{
Tae Kyu Ahn, Soo Kang, Jin Hui Paik, Young Ho Seo \\ Department of Emergency Medicine, Inha University School of Medicine, Incheon, Korea
}

The coronavirus disease 2019 (COVID-19) vaccines are categorized according to the manufacturing technique, including mRNA vaccines and adenovirus vector vaccines. According to previous studies, the reported efficacy of the COVID-19 vaccine is excellent regardless of the type of vaccine, and the majority of studies have shown similar results for safety. Most of the adverse reactions after vaccination were mild or moderate grade, and severe reactions were reported in a very small proportion. However, the adverse reactions that might occur after nationwide vaccinations can contribute to crowding of emergency departments, and this can further lead to significant obstacles to providing necessary treatment for life-threatening conditions. Therefore, as emergency physicians, we would like to present some concerns and suggestions to prevent these predictable problems.

Keywords COVID-19; Vaccination; Adverse effects; Emergency service, hospital

\section{INTRODUCTION}

The first case of coronavirus disease (COVID-19) was reported in late December 2019 in Wuhan, China.' This infectious disease started to spread rapidly across China and then to many countries. The World Health Organization declared COVID-19 as a pandemic in March 2020. Because of the devastating effect of this disease, the development of a vaccine against severe acute respiratory syndrome coronavirus (SARS-CoV)-2, which is the causative virus of COVID-19, has progressed rapidly and extensively. ${ }^{2,3}$

SARS-CoV-2 has a spike protein that induces a host response by binding to the angiotensin converting enzyme 2 receptor, the same receptor used by the SARS-CoV. ${ }^{4}$ Vaccines have been de-

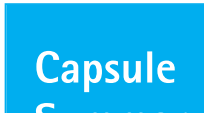

Summary

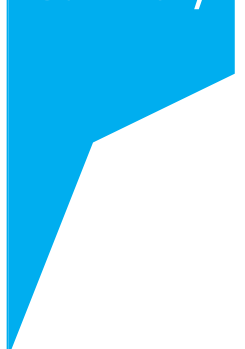

What is already known

The COVID-19 vaccines are categorized into several types. Some previous studies have reported on the safety of the COVID-19 vaccine. Most of the adverse reactions after vaccination were mild or moderate grade, and severe grade was rare.

\section{What is new in the current study}

After nationwide vaccinations, the adverse reactions may lead to crowding of emergency departments. As emergency physicians, we would like to present some concerns and some suggestions to prevent this from happening.
}

Received: 14 April 2021

Revised: 08 June 2021

Accepted: 11 June 2021

Correspondence to: Jin Hui Paik Department of Emergency Medicine, Inha University School of Medicine, 100 Inha-ro, Nam-gu, Incheon 22212, Korea

E-mail: riven2ne@inha.ac.kr ORCID

https://orcid.org/0000-0002-9718-8913

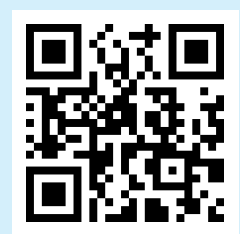

How to cite this article:

Ahn TK, Kang S, Paik JH, Seo YH. Adverse reactions due to coronavirus vaccine and preventive measures recommended by emergency physicians. Clin Exp Emerg Med 2021;8(3):153-159. https://doi. org/10.15441/ceem.21.067

This is an Open Access article distributed under the terms of the Creative Commons Attribution Non-Commercial License (https:// creativecommons.org/licenses/by-nc/4.0/). 
veloped, with the spike protein as the main target, and several vaccines have been administered in various countries.

Currently, several studies on the efficacy and safety of COVID-19 vaccines have been reported. We would like to review the safety of the COVID-19 vaccines reported in those studies and make some suggestions for emergency physicians to help prepare for patients presenting with adverse vaccine reactions.

\section{TYPES OF COVID-19 VACCINES}

COVID-19 vaccines are categorized according to the manufacturing technique: mRNA vaccines, adenovirus vector vaccines, and inactivated virus vaccines. ${ }^{5}$ mRNA vaccines, which are developed by Pfizer-BioNtech, New York, NY, USA (BNT162b2) and Moderna, Cambridge, MA, USA (mRNA-1273), are lipid nanoparticle-encapsulated, nucleoside-modified RNA-based vaccines that encode the receptor-binding domain of the SARS-CoV-2 spike protein. $^{6-9}$ This lipid nanoparticle carrier system prevents the rapid enzymatic degradation of mRNA and facilitates in vivo delivery. ${ }^{6}$ Because lipid nanoparticles are sensitive to temperature, this type of vaccine should be transported and stored under extremely low temperatures. ${ }^{7}$

ChAd0x1 nCoV-19, which is developed by AstraZeneca, London, $U K$, is a replication-defective chimpanzee adenovirus vector vaccine. This vaccine contains the SARS-CoV-2 structural surface glycoprotein antigen (spike protein) gene. ${ }^{10,11}$ Recombinant adenoviruses were initially developed as vehicles for gene therapy. However, they are currently being used as vaccine vectors because of their attractive characteristics. The adenovirus genome can be rendered replication-defective by deleting certain regions, and it can induce transgene-specific immune responses. Additionally, because of its relative thermostability, it is easier to store and transport $\mathrm{ChAdO} \times 1 \mathrm{nCoV}-19$ vaccines than mRNA vaccines. ${ }^{12,13}$ However, preexisting immunity due to natural infections of adenovirus may reduce the immunogenicity of vector vaccines. ${ }^{12}$ Therefore, adenoviruses isolated from chimpanzees, not human adenoviruses, are being used as vaccine carriers. ${ }^{13}$ Previously, replication-defective chimpanzee adenovirus vectors have been used as novel vaccines, such as Ebola vaccines. ${ }^{14,15}$ The Ad26.COV2.S vaccine (Bridgewater Township, NJ, USA) and the Gam-COVID-Vac (Sputnik V; Gamaleya Research Institute, Moscow, Russia) are also recombinant, replication-defective adenovirus vector vaccines that contain the SARS-CoV-2 spike protein. ${ }^{16,17}$

The CoronaVac (Sinovac Biotech, Beijing, China) and Sinopharm COVID-19 vaccines have the inactivated form of SARSCoV-2. ${ }^{18,19}$ Inactivated virus vaccines have been widely used for vaccine development, including polio, hepatitis $A$, and influenza vaccines. ${ }^{20-22}$ In an inactivated virus vaccine, the pathogen is killed or modified so that it cannot cause the disease. CoronaVac is a vaccine that inactivates SARS-CoV-2 by injecting beta-propiolactone after harvesting the virus using African green monkey kidney cells (Vero cells). ${ }^{18}$ The inactivated form of SARS-CoV-2 can no longer replicate, but the spike protein remains intact and can induce immunogenicity.

\section{REPORTED EFFICACY OF COVID-19 VACCINES}

Both humoral and cellular immune responses are critical in verifying the immunity induced by vaccines. ${ }^{5}$ Several previous studies demonstrated the efficacy of BNT162b2 and ChAdOx1 nCoV-19 vaccines in inducing both responses. Sahin et al. ${ }^{23}$ in one of the studies, demonstrated that two doses of BNT162b2 elicited high SARS-CoV-2 neutralizing antibody titers. Additionally, robust receptor-binding domain-specific CD8+ and Thelper type 1 CD4+ T cell responses were elicited by two doses of BNT162b2, and interferon- $\gamma$ was produced by a large fraction of cells. A previous study demonstrated that BNT162b2 vaccination was 95\% effective in preventing the occurrence of COVID-19.?

Vaccination with $\mathrm{ChAdO} \times 1 \mathrm{nCoV}-19$ showed anti-spike protein antibody responses and the induction of antigen-specific $T$ cells against the SARS-CoV-2 spike protein. ${ }^{24,25}$ According to Voysey et al.," ${ }^{11}$ the overall efficacy of ChAdOx1 nCoV-19 was 70.4\%. Interestingly, the efficacy was higher in participants who received a low dose followed by a standard dose (90.0\%) than in those who received two standard doses (62.1\%).

\section{ADVERSE REACTIONS AFTER VACCINATION}

Several adverse reactions due to vaccination have been reported, including hypersensitivity responses and excessive cytokine release. ${ }^{26}$ Hypersensitivity is classified into four types according to the mechanism triggered by vaccines. Both the active component (antigen) and the other components of the vaccine can cause hypersensitivity. ${ }^{27}$ Anaphylaxis is an acute onset systemic reaction that requires urgent management, and it is considered the most serious hypersensitivity reaction. The incidence rate of anaphylaxis after vaccination is estimated to be approximately 1.31 per million vaccine doses. ${ }^{27}$ Proinflammatory cytokines such as interleukin 1, interleukin 6 , and tumor necrosis factor a are released in response to vaccination. ${ }^{26}$ These cytokines can cause pain at the local site by inducing inflammation. ${ }^{28}$ Additionally, they may cause systemic symptoms such as headache, fatigue, malaise, nausea, and fever. ${ }^{28,29}$ Unfortunately, these systemic symptoms are similar 
to the symptoms of infectious diseases including COVID-19.7 Safety reports of COVID-19 vaccines demonstrated in previous studies are summarized in Table 1.,10,11,30-33

\section{Reported safety of BNT162b2 and the associated adverse reactions after vaccination}

A previous study analyzed the data of local and systemic reactions by assessing electronic diaries from 8,183 participants among BNT162b2 recipients. ${ }^{7}$ The participants were divided into two groups according to age (16-55 years of age as younger recipients and $>55$ years of age as older recipients), and the degree of local and systemic reactions was categorized into four grades as shown in Tables 2 and 3, respectively. Both local and systemic reactions were more commonly reported in younger recipients. Mild to moderate pain at the injection site was the most commonly reported local reaction among BNT162b2 recipients, with less than $1 \%$ of participants reporting severe pain. Local reactions resolved within 1-2 days in most cases, and they did not increase after the second dose when compared to that after the first dose. However, a higher proportion of participants had systemic reac- tions after the second dose than after the first dose. Regardless of the age group, the most commonly reported systemic reaction was fatigue. After the second dose, fatigue was reported in 59\% of participants among younger recipients and 51\% among older recipients. Fever (body temperature $\geq 38^{\circ} \mathrm{C}$ ) was reported to occur after the second dose in 16\% and 11\% of younger recipients and older recipients, respectively. Although four serious adverse events were reported, namely, shoulder injury related to vaccine administration, right axillary lymphadenopathy, paroxysmal ventricular arrhythmia, and right leg paresthesia, the incidence was similar to that of the placebo group $(0.6 \%$ and $0.5 \%$, respective-

Table 2. Grade of solicited local adverse reactions

\begin{tabular}{|c|c|c|}
\hline Grade & Pain at the injection site & Redness and swelling \\
\hline Mild & $\begin{array}{l}\text { Does not interfere with } \\
\text { activity }\end{array}$ & $2.0-5.0 \mathrm{~cm}$ in diameter \\
\hline Moderate & Interferes with activity & $5.0-10.0 \mathrm{~cm}$ in diameter \\
\hline Severe & Prevents daily activity & $>10.0 \mathrm{~cm}$ in diameter \\
\hline Grade 4 & $\begin{array}{l}\text { Emergency department } \\
\text { visit or hospitalization }\end{array}$ & $\begin{array}{l}\text { Necrosis or exfoliative dermatitis for } \\
\text { redness and necrosis for swelling }\end{array}$ \\
\hline
\end{tabular}

Table 1. Safety of the COVID-19 vaccines reported in previous studies

\begin{tabular}{|c|c|c|c|c|}
\hline Reference & Design & Vaccine & Safety outcome & Findings \\
\hline $\begin{array}{l}\text { Polack et al. } \\
(2020)^{7}\end{array}$ & $\mathrm{RCT}$ & BNT162b2 & $\begin{array}{l}\text { Local and systemic reac- } \\
\text { tions, serious adverse } \\
\text { events }\end{array}$ & $\begin{array}{l}\text { Mild to moderate pain at the injection site }(66 \%-83 \%) \text {, fatigue }(51 \%-59 \%) \text {, and headache } \\
(39 \%-52 \%) \text {. } \\
\text { Incidence of serious adverse events was similar between the vaccine and placebo groups, no } \\
\text { vaccine related deaths were reported. }\end{array}$ \\
\hline $\begin{array}{l}\text { Walsh et al. } \\
(2020)^{30}\end{array}$ & $\mathrm{RCT}$ & BNT162b2 & $\begin{array}{l}\text { Local and systemic reac- } \\
\text { tions }\end{array}$ & $\begin{array}{l}\text { Mild to moderate pain at the injection site (67\%-92\%), fatigue ( } 25 \%-75 \%) \text {. } \\
\text { Grade } 4 \text { events (all events indicated an emergency department visit or hospitalization) were not } \\
\text { reported for both local and systemic reactions. }\end{array}$ \\
\hline $\begin{array}{l}\text { Shimabukuro } \\
\text { et al. }(2021)^{31}\end{array}$ & Review & $\begin{array}{l}\text { BNT162b2 } \\
\text { mRNA-1273 }\end{array}$ & Anaphylaxis & $\begin{array}{l}\text { Incidence of anaphylaxis was } 4.7 \text { cases/million doses for BNT162b2, and } 2.5 \text { cases/million doses } \\
\text { for mRNA-1273. No deaths from anaphylaxis after vaccination were reported. }\end{array}$ \\
\hline $\begin{array}{l}\text { Folegatti et al. } \\
\qquad(2020)^{33}\end{array}$ & $\mathrm{RCT}$ & ChAdOx nCoV-19 & $\begin{array}{l}\text { Local and systemic reac- } \\
\text { tions, serious adverse } \\
\text { events }\end{array}$ & $\begin{array}{l}\text { Tenderness and pain were the most commonly reported local reactions ( } 83 \% \text { and } 67 \%) \text {, and } \\
\text { fatigue and headache were the most commonly reported systemic reactions (70\% and 68\%). } \\
\text { There were no serious adverse events related to ChAdOx nCoV- } 19 \text {. }\end{array}$ \\
\hline $\begin{array}{l}\text { Ramasamy et al. } \\
(2020)^{10}\end{array}$ & $\mathrm{RCT}$ & ChAdOx nCoV-19 & $\begin{array}{l}\text { Local and systemic reac- } \\
\text { tions, serious adverse } \\
\text { events }\end{array}$ & $\begin{array}{l}\text { Local adverse reactions were reported in } 61 \%-88 \% \text {, and systemic adverse reactions were } \\
\text { reported in } 65 \%-86 \% \text { of participants receiving two standard doses of ChAd0x nCoV- } 19 \text {. } \\
\text { No serious adverse events were considered to be related to the study vaccine. }\end{array}$ \\
\hline $\begin{array}{l}\text { Voysey et al. } \\
\qquad(2021)^{11}\end{array}$ & $\mathrm{RCT}$ & ChAdOx nCoV-19 & Serious adverse events & $\begin{array}{l}\text { Eighty-four serious adverse events were reported in the ChAdOx nCoV-19 recipients, but the in- } \\
\text { cidence rate was similar to the control group ( } 0.7 \% \text { and } 0.8 \% \text {, respectively). }\end{array}$ \\
\hline $\begin{array}{l}\text { Tobaiqy et al. } \\
(2021)^{32}\end{array}$ & Review & ChAdOx nCoV-19 & $\begin{array}{l}\text { Thrombotic adverse reac- } \\
\text { tions }\end{array}$ & $\begin{array}{l}\text { Twenty-eight events were associated with thrombotic adverse reactions among the } 54,571 \\
\text { adverse reaction reports, but no clear causal effect of the vaccine was determined. }\end{array}$ \\
\hline
\end{tabular}

$\mathrm{RCT}$, randomized controlled trial.

Table 3. Grade of solicited systemic adverse reactions

\begin{tabular}{llll}
\hline Grade & \multicolumn{1}{c}{ Fever } & Vomiting & Diarrhea \\
\hline Mild & $38.0^{\circ} \mathrm{C}-38.4^{\circ} \mathrm{C}$ & $1-2$ times in 24 hours & $2-3$ loose stools in 24 hours \\
Moderate & $38.4^{\circ} \mathrm{C}-38.9^{\circ} \mathrm{C}$ & $>2$ times in 24 hours & $4-5$ loose stools in 24 hours \\
Severe & $38.9^{\circ} \mathrm{C}-40.0^{\circ} \mathrm{C}$ & Requires intravenous hydration & $\geq 6$ loose stools in 24 hours \\
Grade 4 & All events indicated an emergency department visit or hospitalization & Some interference with activity & Prevents daily activity \\
\hline
\end{tabular}

a) Fatigue, headache, chills, new or worsened muscle pain, new or worsened joint pain. 
Iy). Two BNT162b2 recipients (total 18,860) and four placebo recipients (total 18,846) died, but none of the deaths were related to vaccine or placebo administration.

Another study on BNT162b2 demonstrated similar results on safety. In the study of Walsh et al., ${ }^{30}$ pain at the injection site was the most commonly reported local reaction, and redness and swelling were less common. Most of the local reactions were mild to moderate grade, and none of the participants reported grade 4 local reactions. Systemic reactions to BNT162b2 included fever, fatigue, headache, chills, vomiting, diarrhea, muscle pain, and joint pain; a higher proportion of participants had systemic reactions after the second dose, except vomiting and diarrhea. The most commonly reported systemic event was fatigue, which was reported in 75\% of participants aged 18 to 55 years and $41.7 \%$ of participants aged 65 to 85 years. Similar to local reactions, most systemic reactions were mild to moderate grade, and grade 4 local reactions were not reported. ${ }^{30}$

After the administration of 9,943,247 doses of BNT162b2, a total of 47 case reports met the Brighton Collaboration case definition criteria for anaphylaxis, and the cases were identified as anaphylaxis. ${ }^{31}$ Among the total patients, 36 (77\%) had a documented history of allergies and 16 (34\%) had a history of anaphylaxis. Of the 7,581,429 recipients of mRNA-1273 vaccines, 19 case reports had reported anaphylaxis. ${ }^{31}$ Sixteen (84\%) patients had a documented history of allergies, and five (26\%) of them had a history of anaphylaxis. Among the 66 patients with anaphylaxis, $32(48 \%)$ were hospitalized, including seven who required endotracheal intubation, and 34 (52\%) were treated in the emergency department (ED). The incidence rates of anaphylaxis after vaccination with mRNA vaccines are 4.7 cases/million doses and 2.5 cases/million doses for BNT162b2 and mRNA-1273 vaccines, respectively. ${ }^{31}$ No deaths due to anaphylaxis were reported. Additionally, another study have reported the occurrence of adverse events affecting the orofacial region including facial palsy, facial swelling, and swollen lip in BNT162b2 and mRNA-1273 recipients. ${ }^{34}$ However, this study have not determined whether they were vaccine-related adverse effects because there is an inconsistency in the results between Europe and North America. ${ }^{34}$

\section{Reported safety of ChAdOx nCoV-19 vaccines and the associated adverse reactions after vaccination}

The most frequently reported local adverse reaction due to vaccination with the ChAdOX $1 \mathrm{nCoV}-19$ vaccine was injection site tenderness, followed by injection site pain. ${ }^{10}$ Most of these reactions occurred within the first two days after vaccination and decreased thereafter. Other local symptoms including induration, itching, redness, swelling, and warmth were observed in very small proportions compared to tenderness and pain. The most frequently reported systemic adverse reaction was fatigue, followed by headache, myalgia, and malaise. Systemic reactions such as chills, fever (body temperature $\geq 38^{\circ} \mathrm{C}$ ), joint pain, and nausea were reported in a relatively small proportion. Both local and systemic reactions have been reported more commonly in the younger age (18 to 55 years) group, and the majority of adverse reactions were mild to moderate in terms of severity. Interestingly, unlike the mRNA vaccine, the presence of systemic adverse reactions after the second dose did not occur at a higher proportion than that after the first dose. ${ }^{10,11,32}$

Another study reported the prophylactic effect of paracetamol on adverse reactions. ${ }^{33}$ Although solicited local and systemic adverse reactions were more common in the ChAdOx $1 \mathrm{nCoV}-19$ group than in the control group, prophylactic paracetamol reduced the frequency of adverse reactions, including pain, fever, chills, headache, and malaise. The most common local adverse reaction was tenderness (77\% and $83 \%$ in the paracetamol group and the no paracetamol group, respectively), followed by pain (50\% and 67\% in the paracetamol group and the no paracetamol group, respectively). Fatigue and headache were the most commonly reported systemic adverse reactions. Fatigue was reported by $340(70 \%)$ participants in the no paracetamol group and by $40(71 \%)$ participants in the paracetamol group, whereas headaches were reported by 331 (68\%) participants in the no paracetamol group and $34(61 \%)$ in the paracetamol group. Fever (body temperature $\geq 38^{\circ} \mathrm{C}$ ) was reported by $87(18 \%)$ and nine $(16 \%)$ participants in the no paracetamol group and the paracetamol group, respectively. The severity and intensity of local and systemic adverse reactions were highest on day 1 after vaccination. However, no patients were hospitalized due to local and systemic adverse reactions.

In one previous study, 84 serious adverse events were reported in 79 of 5,807 participants who were vaccinated with ChAd0x1 nCoV-19. ${ }^{11}$ However, the incidence of these events was similar to that of the control group ( $0.7 \%$ and $0.8 \%$, respectively). Moreover, these adverse events occurred in different systems, including the cardiovascular, nervous, and gastrointestinal systems, as well as infections, and the authors did not demonstrate a consistent pattern clarifying the relationship with the vaccine. Three cases of transverse myelitis were reported as suspected serious adverse reactions due to ChAdO $\times 1 \mathrm{nCoV}-19$ vaccines, but two of them were determined to be unlikely to be related to vaccination. One case of transverse myelitis was considered to be possibly related to vaccination.

From March 11, 2021, several European countries (including Denmark, France, Italy, Latvia, Norway, Spain, Sweden, and The 
Netherlands) temporarily suspended the use of the ChAdOx nCoV19 as a precautionary move after obtaining reports of blood clots and death. ${ }^{35}$ In a previous study conducted using the EudraVigilance database from February 17, to March 12, 2021, a total of 54,571 adverse reactions were reported, and 28 cases were identified as thromboembolic events. ${ }^{32}$ Among the total patients, 19 were female, and two female patients and one male patient died. Most of the thromboembolic events reported were deep vein thrombosis and pulmonary thromboembolism, while there were two cases of pelvic vein thrombosis and one case each of cerebral venous sinus thrombosis, carotid artery thrombosis, and thrombophlebitis. However, to verify the relationship between the occurrence of adverse reactions and vaccine administration, further studies that include the natural incidence of thromboembolic events and patients' characteristics including risk factors are needed. It is not yet possible to conclude that these thromboembolic events are related to vaccine administration. As a result, the European Medicines Agency and the World Health Organization have stated that there is no indication that vaccination is linked to thromboembolic events. ${ }^{32,35}$

\section{SUMMARY AND SUGGESTIONS}

COVID-19 vaccination is a key issue and challenge in global health. The reported efficacy of various COVID-19 vaccines is excellent regardless of the type of vaccine, and the majority of studies have shown similar results for safety. Most of the local and systemic adverse reactions after vaccination have been of mild or moderate grade, and severe reactions have been reported in a very small proportion of patients. In general, these adverse reactions tended to be more common and more severe in younger age groups than in older age groups. In the study of BNT162b2 vaccines, more systemic reactions were reported after the second dose than after the first dose. The most common local adverse reaction was tenderness and pain at the injection site, and most patients improved within a few days. As a systemic adverse reaction, fatigue was most commonly reported, with headache and myalgia also commonly reported. Although fever (body temperature $\geq 38^{\circ} \mathrm{C}$ ) was reported in approximately $10 \%$ to $20 \%$, most of the systemic adverse reactions resolved within a few days. In addition, except for cases of hospitalization due to anaphylaxis, no patients required hospitalization due to adverse reactions with a proven relationship to vaccine administration.

Since the emergence of the COVID-19 pandemic, there have been many changes in medical institutions including the ED. ${ }^{36}$ In particular, all types of medical personnel are making intense efforts to prevent the spread of COVID-19 in medical institutions.
In the ED, patients with suspected COVID-19 are isolated, and medical personnel are required to wear personal protective equipment (PPE) when being in close contact with the patient. ${ }^{37,38}$ The need for patient isolation and the additional amount of time required to wear PPE have caused many difficulties not only for medical personnel but also for patients. ${ }^{38,39}$ Moreover, the inadequate bed capacity for isolation has also been an important issue, which increases the burden on the emergency medical system. ${ }^{40}$ As mentioned earlier, the systemic adverse reactions that occur after COVID-19 vaccination are similar to those of infectious diseases including COVID-19. Therefore, with the current guidelines for determining the need for isolation based on symptoms, it is inevitable that patients who visit the ED with complaints of a systemic adverse reaction will also be isolated. Although there is no risk of transmission among these patients and they do not require special measures such as isolation and wearing PPE, it cannot be easily determined that it is a systemic reaction caused only by vaccination. In particular, the Republic of Korea experienced the spread of Middle East respiratory syndrome in medical institutions in 2015, and the EDs were considered a high-risk place for disease transmission. ${ }^{41}$ Based on these past experiences and the nature of EDs visited by various people, all types of emergency medical personnel are bound to be more sensitive to the prevention of disease transmission. In the near future, nationwide vaccinations will be carried out to the general population. If more patients visit the ED with complaints of adverse reactions, then the aforementioned problems, including patient isolation and treatment time will increase. Moreover, these reactions may contribute to ED crowding, which is known to have a negative impact on patients, such as a high mortality rate. ${ }^{42,43}$ This can further lead to significant obstacles to providing necessary treatment for patient with life-threatening conditions.

It is difficult to manage these patients because urgent treatment may be required, such as for anaphylaxis, or symptoms may be caused by other diseases not related to the vaccine administration. However, it is clear that the number of patients with adverse reactions will increase after nationwide vaccination. Therefore, we would like to make some suggestions for the prevention of predictable problems such as increasing treatment time and ED crowding. First, it will be necessary to increase isolation bed capacity. Given that adverse reactions will occur after nationwide vaccination, each medical institution and ED will require more isolation beds than that available currently. If there are inadequate isolation beds, then the patient's treatment may be delayed, which will lead to ED crowding and may negatively affect patient outcomes. Second, a dedicated treatment center for adverse reactions due to vaccine administration that is open $24 / 7$ is 
required. This can help prevent ED crowding and aid clinicians in systematically managing adverse reactions due to vaccine administration. Third, it is possible to consider home observation for a few days or outpatient treatment rather than visiting the ED for most of the adverse reactions after vaccination. According to previous studies on COVID-19 vaccination, most of the local and systemic reactions after vaccination were mild to moderate in severity and improved within a few days. In addition, the use of prophylactic paracetamol may help relieve symptoms. ${ }^{33}$ Therefore, we can consider prescribing an analgesic such as paracetamol to relieve or prevent adverse reactions.

In health care, proper operation is as crucial as knowledge and technology. Therefore, with advances in knowledge and technology, the operation of the health care system must also develop. As there are many studies on the impact of COVID-19 on the social, economic, and public health, studies on vaccination adverse effects, and the resulting changes in health care will also be needed. These studies will provide a blueprint for the proper operation of the ED and medical institutions when new infectious disease outbreaks occur.

\section{CONFLICT OF INTEREST}

No potential conflict of interest relevant to this article was reported.

\section{REFERENCES}

1. Zhu N, Zhang D, Wang W, et al. A novel coronavirus from patients with pneumonia in China, 2019. N Engl J Med 2020;382: 727-33.

2. Krammer F. SARS-CoV-2 vaccines in development. Nature 2020;586:516-27.

3. Voysey M, Costa Clemens SA, Madhi SA, et al. Single-dose administration and the influence of the timing of the booster dose on immunogenicity and efficacy of ChAdO $\times 1 \mathrm{nCoV}-19$ (AZD1222) vaccine: a pooled analysis of four randomised trials. Lancet 2021;397:881-91.

4. Zhou $P$, Yang $X L$, Wang $X G$, et al. A pneumonia outbreak associated with a new coronavirus of probable bat origin. Nature 2020;579:270-3.

5. Brisse $M$, Vrba SM, Kirk N, Liang Y, Ly H. Emerging concepts and technologies in vaccine development. Front Immunol 2020; 11:583077.

6. Castells MC, Phillips EJ. Maintaining safety with SARS-CoV-2 vaccines. N Engl J Med 2021;384:643-9.

7. Polack FP, Thomas SJ, Kitchin N, et al. Safety and efficacy of the BNT162b2 mRNA COVID-19 vaccine. N Engl J Med 2020; 383:2603-15.

8. Sahin U, Muik $A$, Derhovanessian $E$, et al. COVID-19 vaccine BNT162b1 elicits human antibody and TH1 T cell responses. Nature 2020;586:594-9.

9. Jackson LA, Anderson EJ, Rouphael NG, et al. An mRNA vaccine against SARS-CoV-2: preliminary report. N Engl J Med 2020;383:1920-31.

10. Ramasamy MN, Minassian AM, Ewer KJ, et al. Safety and immunogenicity of $\mathrm{ChAdO} \times 1 \mathrm{nCoV}-19$ vaccine administered in a prime-boost regimen in young and old adults (COVOO2): a single-blind, randomised, controlled, phase $2 / 3$ trial. Lancet 2021;396:1979-93.

11. Voysey M, Clemens SAC, Madhi SA, et al. Safety and efficacy of the ChAd0x1 nCoV-19 vaccine (AZD1222) against SARSCoV-2: an interim analysis of four randomised controlled trials in Brazil, South Africa, and the UK. Lancet 2021;397:99111.

12. Tatsis $\mathrm{N}$, Ertl HC. Adenoviruses as vaccine vectors. Mol Ther 2004;10:616-29.

13. Dicks MD, Spencer AJ, Edwards NJ, et al. A novel chimpanzee adenovirus vector with low human seroprevalence: improved systems for vector derivation and comparative immunogenicity. PLoS One 2012;7:e40385.

14. Milligan ID, Gibani MM, Sewell $R$, et al. Safety and immunogenicity of novel adenovirus type 26- and modified vaccinia Ankara-Vectored Ebola vaccines: a randomized clinical trial. JAMA 2016;315:1610-23.

15. Ledgerwood JE, DeZure AD, Stanley DA, et al. Chimpanzee adenovirus vector Ebola vaccine. N Engl J Med 2017;376:92838 .

16. Sadoff J, Gray G, Vandebosch A, et al. Safety and efficacy of single-dose Ad26.COV2.S vaccine against COVID-19. N Engl J Med 2021;384:2187-201.

17. Logunov DY, Dolzhikova IV, Shcheblyakov DV, et al. Safety and efficacy of an rAd26 and rAd5 vector-based heterologous primeboost COVID-19 vaccine: an interim analysis of a randomised controlled phase 3 trial in Russia. Lancet 2021;397:671-81.

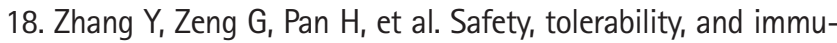
nogenicity of an inactivated SARS-CoV-2 vaccine in healthy adults aged 18-59 years: a randomised, double-blind, placebo-controlled, phase 1/2 clinical trial. Lancet Infect Dis 2021; 21:181-92.

19. Xia $S$, Duan $K$, Zhang $Y$, et al. Effect of an inactivated vaccine against SARS-CoV-2 on safety and immunogenicity outcomes: interim analysis of 2 randomized clinical trials. JAMA 2020; 324:951-60. 
20. Patel M, Zipursky S, Orenstein W, Garon J, Zaffran M. Polio endgame: the global introduction of inactivated polio vaccine. Expert Rev Vaccines 2015;14:749-62.

21. Andre F, Van Damme P, Safary A, Banatvala J. Inactivated hepatitis $A$ vaccine: immunogenicity, efficacy, safety and review of official recommendations for use. Expert Rev Vaccines 2002; 1:9-23.

22. Vellozzi C, Burwen DR, Dobardzic $A$, Ball R, Walton $K$, Haber P. Safety of trivalent inactivated influenza vaccines in adults: background for pandemic influenza vaccine safety monitoring. Vaccine 2009;27:2114-20.

23. Sahin U, Muik A, Vogler I, et al. BNT162b2 induces SARS-CoV2-neutralising antibodies and T cells in humans. medRxiv 2020. 12.09.20245175 [Preprint]. 2020 [cited 2021 Apr 13]. Available from: https://doi.org/10.1101/2020.12.09.20245175.

24. Barrett JR, Belij-Rammerstorfer $S$, Dold $C$, et al. Phase $1 / 2$ trial of SARS-CoV-2 vaccine ChAdOx1 nCoV-19 with a booster dose induces multifunctional antibody responses. Nat Med 2021;27:279-88.

25. Ewer KJ, Barrett JR, Belij-Rammerstorfer S, et al. T cell and antibody responses induced by a single dose of ChAdOx $1 \mathrm{nCoV}$ 19 (AZD1222) vaccine in a phase 1/2 clinical trial. Nat Med 2021;27:270-8.

26. Roth JA. Mechanistic bases for adverse vaccine reactions and vaccine failures. In: Roth JA. Veterinary vaccines and diagnostics. [place unknown]: Elsevier; 1999. p.681-700.

27. McNeil MM, DeStefano F. Vaccine-associated hypersensitivity. J Allergy Clin Immunol 2018;141:463-72.

28. Christian LM, Porter K, Karlsson E, Schultz-Cherry S. Proinflammatory cytokine responses correspond with subjective side effects after influenza virus vaccination. Vaccine 2015; 33:3360-6.

29. Dantzer R, Kelley KW. Twenty years of research on cytokineinduced sickness behavior. Brain Behav Immun 2007;21:153-60.

30. Walsh EE, Frenck RW Jr, Falsey AR, et al. Safety and immunogenicity of two RNA-based COVID-19 vaccine candidates. N Engl J Med 2020;383:2439-50.

31. Shimabukuro T, Cole M, Su JR. Reports of anaphylaxis after receipt of mRNA COVID-19 vaccines in the US: December 14, 2020-January 18, 2021. JAMA 2021;325:1101-2.

32. Tobaiqy M, Elkout $H$, MacLure K. Analysis of thrombotic ad- verse reactions of COVID-19 AstraZeneca vaccine reported to EudraVigilance Database. Vaccines (Basel) 2021;9:393.

33. Folegatti PM, Ewer KJ, Aley PK, et al. Safety and immunogenicity of the ChAdOx1 nCoV-19 vaccine against SARS-CoV-2: a preliminary report of a phase $1 / 2$, single-blind, randomised controlled trial. Lancet 2020;396:467-78.

34. Cirillo N. Reported orofacial adverse effects of COVID-19 vaccines: the knowns and the unknowns. J Oral Pathol Med 2021; 50:424-7.

35. Wise J. COVID-19: European countries suspend use of OxfordAstraZeneca vaccine after reports of blood clots. BMJ 2021;372: n699.

36. Boyle AA, Henderson K. COVID-19: resetting ED care. Emerg Med J 2020;37:458-9.

37. Af Ugglas B, Skyttberg N, Wladis A, Djarv T, Holzmann MJ. Emergency department crowding and hospital transformation during COVID-19, a retrospective, descriptive study of a university hospital in Stockholm, Sweden. Scand J Trauma Resusc Emerg Med 2020;28:107.

38. O'Reilly GM, Mitchell RD, Mitra B, et al. Impact of patient isolation on emergency department length of stay: a retrospective cohort study using the Registry for Emergency Care. Emerg Med Australas 2020;32:1034-9.

39. Smereka J, Szarpak L. The use of personal protective equipment in the COVID-19 pandemic era. Am J Emerg Med 2020; 38:1529-30.

40. Fan EM, Nguyen NHL, Ang SY, et al. Impact of COVID-19 on acute isolation bed capacity and nursing workforce requirements: a retrospective review. J Nurs Manag 2021 Jan 22 [Epub]. https://doi.org/10.1111/jonm.13260.

41. Cho SY, Kang JM, Ha YE, et al. MERS-CoV outbreak following a single patient exposure in an emergency room in South Korea: an epidemiological outbreak study. Lancet 2016;388:9941001.

42. Sprivulis PC, Da Silva JA, Jacobs IG, Frazer AR, Jelinek GA. The association between hospital overcrowding and mortality among patients admitted via Western Australian emergency departments. Med J Aust 2006;184:208-12.

43. Hoot NR, Aronsky D. Systematic review of emergency department crowding: causes, effects, and solutions. Ann Emerg Med 2008;52:126-36. 